\title{
Comparison of Pre/Post-Operative CT Image Volumes to Preoperative Digitization of Partial Hepatectomies: A Feasibility Study in Surgical Validation
}

\author{
Prashanth Dumpuri ${ }^{1}$, Logan W. Clements ${ }^{2}$, Rui Li $^{3}$, Jonathan M. Waite ${ }^{2}$, James D. Stefansic ${ }^{2}$, \\ David A. Geller ${ }^{4}$ M.D., Michael I. Miga ${ }^{1}$, Benoit M. Dawant ${ }^{31}$ \\ ${ }^{1}$ Vanderbilt University, Department of Biomedical Engineering, Nashville, TN 37235 \\ ${ }^{2}$ Pathfinder Therapeutics, Inc., Nashville, TN 37204 \\ ${ }^{3}$ Vanderbilt University, Department of Electrical Engineering and Computer Science, Nashville, \\ TN 37235 \\ ${ }^{4}$ University of Pittsburgh Medical Center Liver Cancer Center, Pittsburgh, PA 15213
}

\begin{abstract}
Preoperative planning combined with image-guidance has shown promise towards increasing the accuracy of liver resection procedures. The purpose of this study was to validate one such preoperative planning tool for four patients undergoing hepatic resection. Preoperative computed tomography (CT) images acquired before surgery were used to identify tumor margins and to plan the surgical approach for resection of these tumors. Surgery was then performed with intraoperative digitization data acquire by an FDA approved image-guided liver surgery system (Pathfinder Therapeutics, Inc., Nashville, TN). Within 5-7 days after surgery, post-operative CT image volumes were acquired. Registration of data within a common coordinate reference was achieved and preoperative plans were compared to the postoperative volumes. Semi-quantitative comparisons are presented in this work and preliminary results indicate that significant liver regeneration/hypertrophy in the postoperative CT images may be present post-operatively. This could challenge pre/post operative CT volume change comparisons as a means to evaluate the accuracy of preoperative surgical plans.
\end{abstract}

Keywords: Image-guided liver surgery, Preoperative planning, Image registration, Liver resection

\section{INTRODUCTION}

For people diagnosed with liver cancer, hepatic resection has been shown to offer the best chance for cure or longterm survival. Accurate tumor-free resection margins combined with the preservation of healthy liver parenchyma is essential for long-term survival of patients after resection of liver tumors. More often than not the surgeons operate intuitively relying on their anatomical knowledge and sense of touch and orientation. Liver vasculature and the relative position of the tumor to important liver vessels make it harder for the surgeon to obtain tumor-free resection margins and highlight the importance of preoperative planning. Preoperative plans are based on preoperative diagnostic images and provide a three-dimensional (3D) visualization of the organ (liver and tumor) and hepatic vasculature along with patient-specific resection plans based on mathematical analyses. Different approaches have been used for this preoperative planning procedure. Soler et al. [1] developed a fully automatic segmentation of the liver with delineation of important anatomical (liver segments), pathological (lesions and tumors) and functional structures (hepatic vessels) and displayed 3D views of the same to improve the planning of hepatic surgery. In addition to displaying the relevant structures [2] developed a virtual reality based computer interface to manipulate the organ and to define the surgical resection planes according to the relevant anatomical and functional structures. In addition to CT scans, [3] used a 3D ultrasound device to assist the surgeon in preoperative planning. Regardless of the preoperative planning method, these studies along with others $[\mathbf{4}, \mathbf{5}]$ have shown that preoperative planning using three-dimensional patient-specific models facilitates the selection of optimal resection path and also improves the accuracy of resection margins. In accordance with this, this paper is concerned with evaluation of software tools developed by Pathfinder Therapeutics Inc. which is dedicated to image-based computer assistance in liver surgery.

\footnotetext{
${ }^{1}$ Michael I. Miga and Benoit M. Dawant are co-founders of Pathfinder Therapeutics Inc.
} 
One such software tool, PlaniSight, allows the surgeon to visualize the three-dimensional surface of the liver by incorporating the patient's individual anatomy obtained from preoperative CT images, thereby allowing the surgeon to plan an optimal resection path based on realistic anatomic conditions. We retrospectively evaluated these preoperative plans for patients undergoing partial hepatectomies at the University of Pittsburgh Medical Center using postoperative CT images acquired 5-7 days after surgery. Surface registrations were performed between surfaces acquired using preoperative and postoperative images; registration results have been presented below as qualitative overlays. Volume comparisons were also made between the preoperative and postoperative surfaces to evaluate the preoperative plans.

\section{METHODS}

\subsection{Patients}

Four patients (all female) with a median age of 57.5 years undergoing hepatic tumor resection therapies were used in this study. All patients were enrolled after obtaining written informed consent for participation in this study which was approved by the University of Pittsburgh institutional review board. Table 1 shows the age (column 2), gender (column 3) and, the type of tumor (column 4) for the patients used in this work. Preoperative and postoperative CT tomograms were acquired for all 4 and postoperative tomograms were typically acquired either 5 or 7 days after surgery. Triphasic sets of CT scans were acquired for both sets of images.

Table 1. Patient Information

\begin{tabular}{|c|c|c|l|}
\hline Patient \# & Age & Gender & \multicolumn{1}{|c|}{ Type of underlying tumor } \\
\hline 1 & 53 & F & Metastases of gastrointestinal stroma tumor \\
\hline 2 & 80 & F & Metastases of lung cancer \\
\hline 3 & 47 & F & Metastases of anal cancer \\
\hline 4 & 62 & F & Metastases of colon cancer \\
\hline
\end{tabular}

\subsection{Image Segmentation, Visualization and Intervention Planning}

PlaniSight was used to accomplish the tasks of image segmentation and analysis. The steps below list the standardized workflow for preoperative planning based on the patient's preoperative 2D CT images:

1. Segmentation of liver and tumor. A level set based technique to segment the organ with minimal user interaction was used to segment the liver and tumor from the abdominal CT scan. After segmenting the normal and diseased liver tissue, a 3D surface is reconstructed using the marching cubes algorithm [6] and smoothed using the FastRBF tool kit (http://www.farfieldtechnology.com). Total liver volume (TLV) is calculated from this step.

2. Segmentation of the vessel tree. Region growing and thresholding techniques have been implemented in the software to segment the liver vasculature. By placing seeds on the hepatic and portal vessel structures individually, the software tool allows for the separation of the vessel systems. Similar to the liver tissue, 3D surfaces of the vessel structures are constructed.

3. Virtual resection. PlaniSight allows for the surgeon to draw virtual transection lines on the CT image data on several slices and these are then interpolated and displayed on the three-dimensional views. The software tool renders the liver transparent thereby allowing the surgeon to precisely determine tumor location and the vicinity to vessels when delineating the transection line and also calculates the distance between the transection line and the closest tumor margin, thereby allowing the surgeon to modify the virtual resection line if necessary. Functional residual liver volume (FLR) is determined from this step, as the liver volume remaining after virtual resection. This volume is sometimes also referred to as the functional remnant liver volume. 
Figure 1 shows the image segmentation, visualization of the segmentation and the virtual resection planes for patient 1 reported in this study. Figures 1A, 1B and 1C show the three views of the patient's preoperative CT image with the

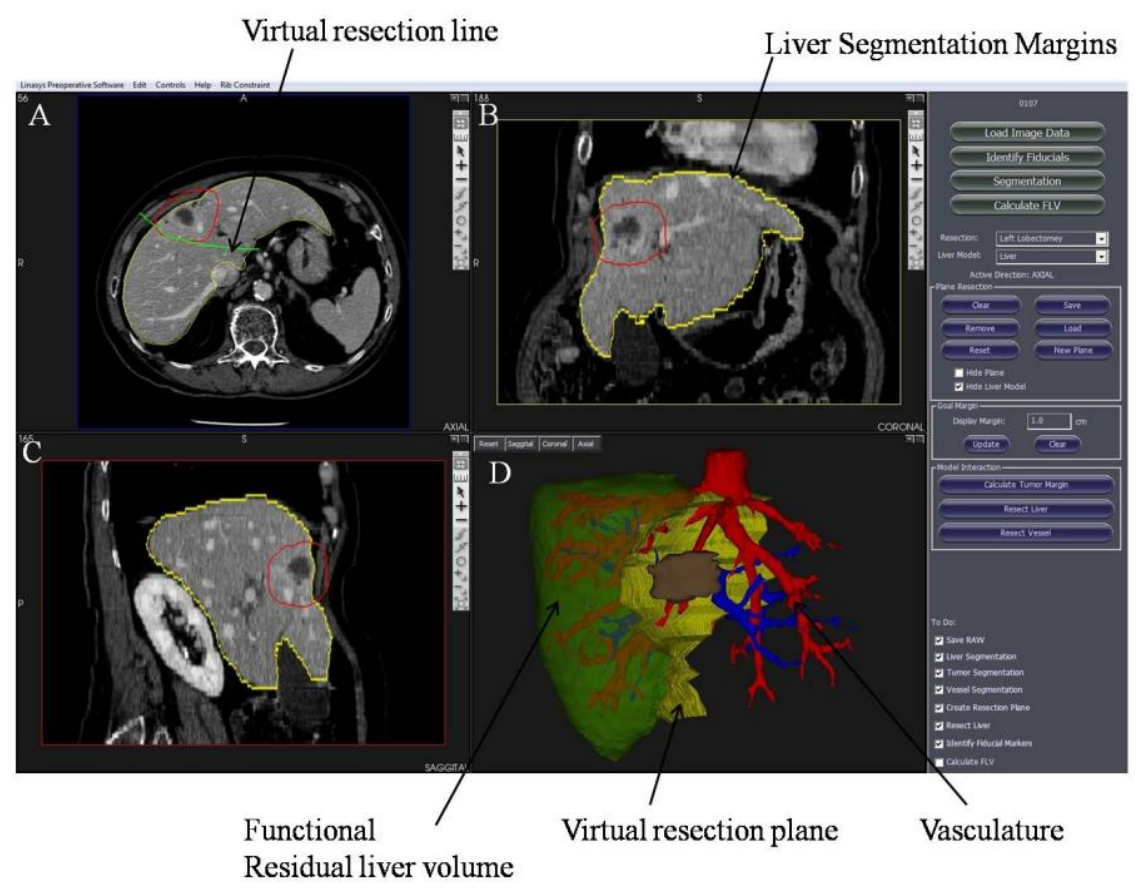

Figure1. (Color online) PreOperative planning. Figures 1A, 1B and 1c show the three views of the patient's preoperative diagnostic CT images. It should be noted that the tumor margins highlighted in these three figures do not represent the actual segmentation of the diseased tissue. Bigger margins have been drawn for better clarity and visual aid. Figure 1D shows the three dimensional surfaces of the structures of interest.

liver segmentation margins. The tumor margins have also been highlighted in this figure for better understanding. It should be noted that the margins shown in the figure do not represent the actual tumor size used for intervention planning. Three-dimensional surface views of the tumor and vessel structures have been shown in Figure 1D. Figure $1 \mathrm{~A}$ also shows the resection line drawn by the surgeon on an axial slice. Similar lines were drawn on successive slices and these lines were interpolated to obtain the resection plane and functional residual liver volume shown in Figure 1D.

Figure 2A shows the 3D surface obtained from preoperative CT images for patient 3 reported in this study. The virtual resection plane shown in this figure was obtained by interpolating the resection lines drawn on CT image slices. Figure $2 \mathrm{~B}$ shows the functional residual liver volume for the same patient. This was obtained by subtracting the resected volume from the total liver volume.

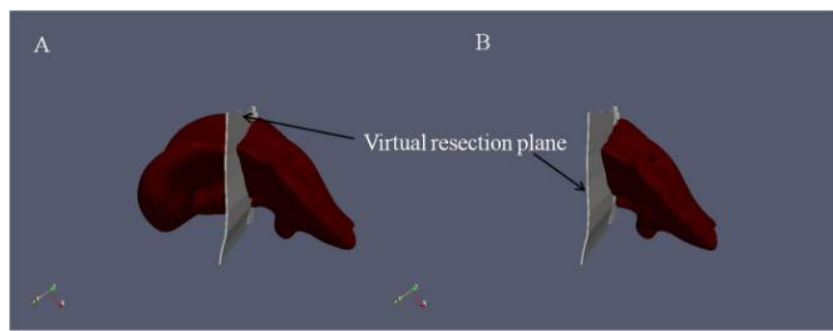

Figure 2. (Color online) Total liver volume (2A) and functional liver volume (2B) for patient 3 reported in this study. Virtual resection plane was obtained by interpolating the resection lines drawn by the surgeon on the preoperative 2D CT slices and was used to calculate the functional liver volume.

\subsection{Registering the Postoperative Images to Preoperative Images}

Partial hepatectomies were then performed by the surgeon. As described earlier, postoperative CT images were acquired 5-7 days after surgery. Liver tissue was segmented from the postoperative images using PlaniSight and a three-dimensional surface was constructed in a manner similar to the method used for preoperative images. The 
postoperative and preoperative surfaces were registered to each other using the iterative closest point (ICP) method [7]. Qualitative and quantitative results are presented in the next section.

\section{RESULTS}

Figure 3 shows qualitatively the results obtained by registering the patient's postoperative 3D surfaces (shown as solid surfaces in the figures) to the preoperative CT surfaces (transparent surfaces). The two surfaces have been overlaid on each other. Figures 3A and 3B (top row) show the overlays for the patient 1 and Figures 3C and 3D the overlays for patient 3 reported in this study.

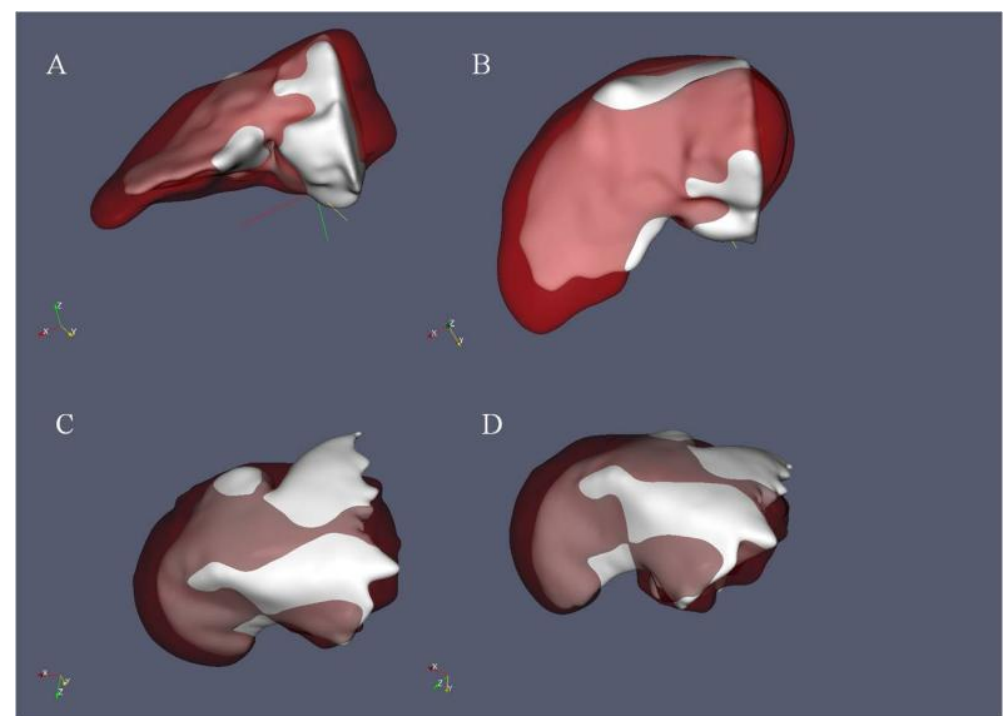

Figure 3. (Color online) Qualitative registration results between the patient's postoperative 3D surfaces and the preoperative surfaces. The registered postoperative 3D surfaces (solid surfaces) have been overlaid on the preoperative 3D surfaces (transparent surfaces). Top row (figures 3A and 3B) shows the overlays for Patient 1 and the bottom row (figures 3C and 3D) the overlays for Patient 3 reported in this study. Two different views of the same results have been shown for both patients for better visual aid and clarity.

Table 2 shows the volumes computed for the total preoperative liver tissue, functional residual liver tissue and the postoperative liver. As stated earlier, functional residual liver tissue refers to the tissue remaining based on the resection lines drawn by the surgeon on the preoperative CT images (in other words the preoperative plan). Relative $\%$ change in volume reported in column 6 of the table was calculated as follows: relative change in volume between postoperative volume and residual volume $=($ Postoperative volume - Functional Residual Liver volume $) /$ PostOp volume $* 100$

Table 2. Total preoperative liver volume, functional residual liver volume obtained from the preoperative plans delineated by the surgeon and the postoperative liver volume for all four patietnts reported in this study.

\begin{tabular}{|c|c|c|c|c|c|}
\hline Patient \# & $\begin{array}{c}\text { PreOp Volume } \\
\left(\mathrm{cm}^{3}\right)\end{array}$ & $\begin{array}{c}\text { Functional } \\
\text { Residual Liver } \\
\text { volume based } \\
\text { on preoperative } \\
\text { plans } \\
\left(\mathrm{cm}^{3}\right)\end{array}$ & $\begin{array}{c}\text { \# of days after } \\
\text { surgery when } \\
\text { PostOp CT scan } \\
\text { was acquired }\end{array}$ & $\begin{array}{c}\text { PostOp } \\
\text { volume } \\
\left(\mathrm{cm}^{3}\right)\end{array}$ & $\begin{array}{c}\text { Relative \% change in } \\
\text { volume between postop } \\
\text { volume and residual } \\
\text { volume } \\
\left(\mathrm{cm}^{3}\right)\end{array}$ \\
\hline 1 & 1970 & 855 & 5 & 1170 & 26.9 \\
\hline 2 & 1081 & 394 & 5 & 437 & 21.2 \\
\hline 3 & 2590 & 1190 & 5 & 1511 & 23.3 \\
\hline 4 & 1880 & 788 & 7 & 1028 & \\
\hline
\end{tabular}




\section{DISCUSSION}

The question as to how to validate image-guided surgery is a persistent challenge within the field. In organs such as the liver, it is difficult to identify soft-tissue targets that are both identifiable on preoperative image volumes and within the operating room. As image-guidance is translated outside of neuro-applications, this challenge will continue and strategies to answer this call must be developed. In this work, a commercial software tool and guidance system (Pathfinder Therapeutics, Inc. - PTI) is evaluated within the context of an approach to validate surgical performance. There are two distinct phases to this clinical evaluation: preoperative planning and imageguidance within the operating room. Preoperative plans are obtained using a software tool developed by PTI. These plans generate three-dimensional surfaces of the diseased and normal liver tissue and liver vasculature and virtual transection/resection lines delineated by the surgeon, which indicate the planned surgical approach. Functional residual liver volume is calculated from the virtual transaction lines. Postoperative CT tomograms were used to validate the planned preoperative resection lines and this report presents a pilot study of 4 patients from the ongoing clinical trial. As stated earlier, the postoperative surfaces were registered to the preoperative ones using a rigid surface-based registration technique. Since the functional remnant liver volumes were obtained using the preoperative plans assuming that the surgeon followed the preoperative resection lines and the plan, the postoperative liver volume should equal the functional remnant liver volume. However, results presented in Table 2 indicate that the postoperative liver volume is greater than the functional remnant liver volume. Figure 4 shows the registered postoperative surface (light transparent surface) overlaid on the functional liver volume (dark transparent surface) along with the planned resection plane for Patients 1 (top row, Figure 4A and 4B) and 2 (bottom row, Figures 4C and 4D) reported in this study. If the surgeon followed the preoperative planning, the postoperative volume should be equal to the functional residual liver volume. However Figure 4 shows that the postoperative liver margins do not line up with the preoperative plans indicating that the postoperative volume is greater than the functional residual liver volume. A similar increase in volume was observed in all four patients as is evident from column 6 in Table 2. Assuming that the surgeon closely followed the preoperative resection plans, this increase in volume suggests significant regeneration in the postoperative scans. Liver regeneration of the residual liver has been documented with almost near complete regeneration at approximately 1 year $[\mathbf{8}, \mathbf{9}]$. However we have observed up to $27 \%$ regeneration 5-7 days after hepatic resection. Though a more detailed analysis is needed, preliminary results suggest that there is significant regeneration 5-7 days after surgery. Other possible contributions such as changes in pressure-volume compliance due to organ perfusion changes may also be important. In any case, this makes it difficult in evaluating the preoperative plans using postoperative CT scans. Based on these preliminary findings, the data collection protocol for the clinical trial has been modified to acquire postoperative CT scans 2 days after surgery where feasible. It should be noted that though the postoperative surfaces were registered to the preoperative liver volumes (i.e., the total liver volumes) similar registration results and volumes were observed when the postoperative surfaces were registered to the functional residual/remnant preoperative surfaces.

It is worth noting a few limitations of this study. Postoperative surfaces were rigidly registered to the preoperative surfaces using a surface-based registration technique. However, recent studies [10, 11] have shown that the liver deforms in a non-rigid manner during hepatic resection therapies. Possible sources of intraoperative deformation include the surgeon removing the surrounding ligaments to better visualize and inspect the organ, the surgeon "packing" the organ for better access to the tumor, gravitational forces causing the liver to sag, and changes in organ perfusion rates. We also observed significant shape changes in the postoperative surfaces, leading us to believe that the liver is still in its deformed state when the postoperative CT scans were acquired. Ligaments that are removed during the surgery are not re-attached to the liver after tumor removal. We hypothesize that this along with the changed perfusion rate due to tumor removal causes the liver to remain deformed in the postoperative scans. This calls for a non-rigid registration technique similar to the one reported in [12]. Also, all four patient cases reported in this study were females with metastatic tumors. More cases with diverse underlying tumor types have to be analyzed before making any significant conclusions. 


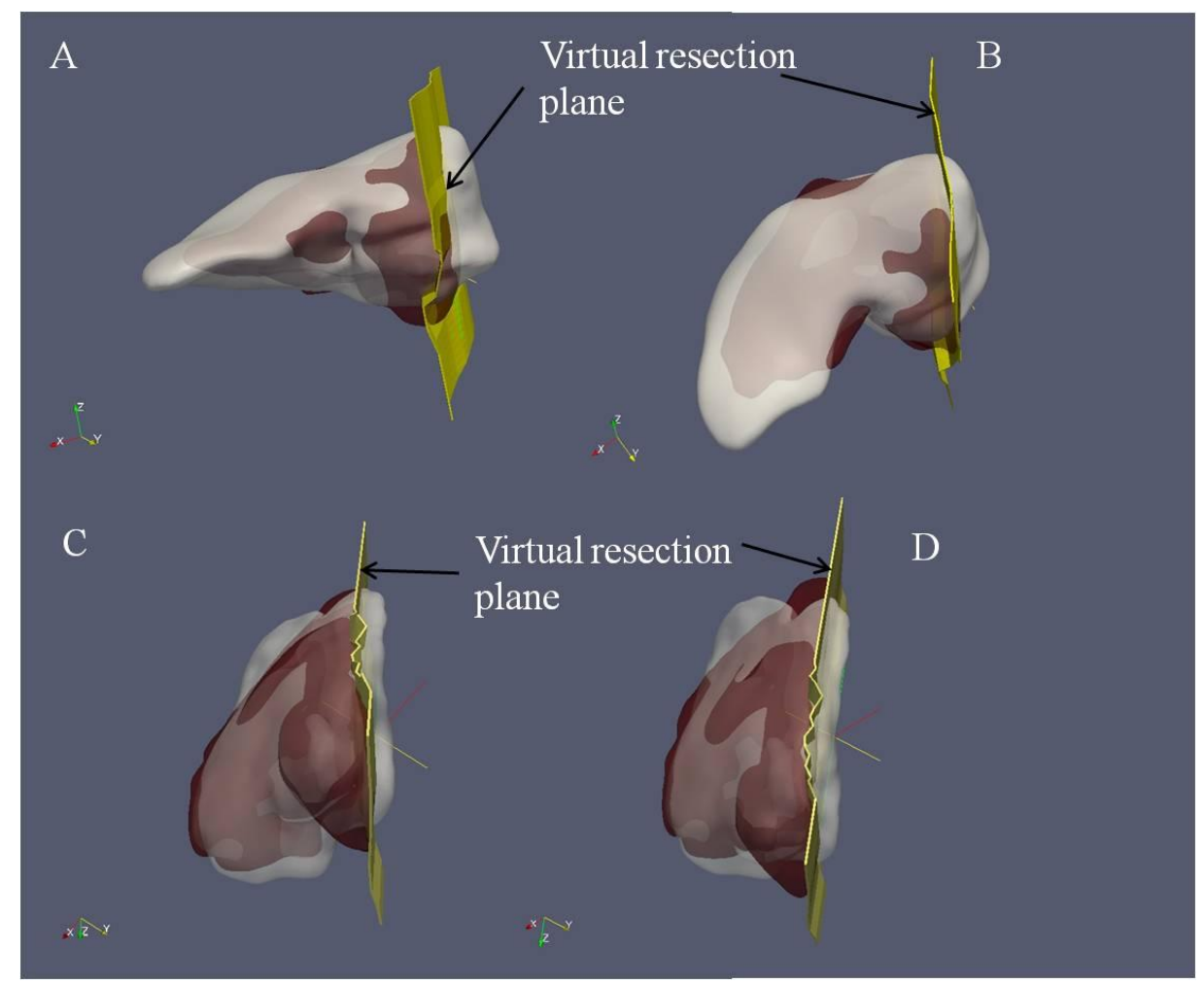

Figure 4. Registration results for Patients 1 (top row, Figure 4A and 4B) and 2 (bottom row, Figures 4C and 4D) reported in this study. Registered postoperative liver volume (light transparent surface) has been overlaid on the functional remnant/residual liver volume (dark transparent surface). This functional residual volume and virtual resection plane were obtained from the patient's preoperative plan. Two different camera angles have been shown for Patient 2 for better visual aid and clarity. Postoperative liver margins do not line up with the preoperative plans indicating that the postoperative volume is greater than the functional residual liver volume.

\section{CONCLUSIONS}

These preliminary results indicate that postoperative CT surfaces acquired 5-7 days after surgery cannot be used to validate the preoperative resection plans. We are working on using the vasculature and the vessel information in preoperative and postoperative $\mathrm{CT}$ images to validate the preoperative resection plans.

\section{ACKNOWLEDGEMENTS}

This work has been supported by National Institute of Health, Grant \# R21 EB007694-01 and National Cancer Institute Grant \# CA119502. The authors would like to thank Dr. Robert L. Galloway for his valuable inputs and would also to thank the radiology and operating room staff at University of Pittsburgh Medical Center for their assistance in data collection. 


\section{REFERENCES}

[1] L. Soler, H. Delingette, G. Malandain, J. Montagnat, N. Ayache, C. Koehl, O. Dourthe, B. Malassagne, M. Smith, D. Mutter, and J. Marescaux, "Fully automatic anatomical, pathological, and functional segmentation from ct scans for hepatic surgery." Comput Aided Surg, vol. 6, no. 3, pp. 131-142, 2001. [Online]. Available: http://dx.doi.org/10.1002/igs.1016

[2] J. Marescaux, J. Clement, V. Tassetti, C. Koehl, S. Cotin, Y. Russier, D. Mutter, H. Delingette, and N. Ayache, "Virtual reality applied to hepatic surgery simulation: the next revolution," Ann.Surg., vol. 228, no. 5, pp. 627-634, Nov 1998. [Online]. Available: PM:9833800

[3] S. Eulenstein, T. Lange, M. Hünerbein, P.-M. Schlag, and H. Lamecker, "Ultrasound-based navigation system incorporating preoperative planning for liver surgery," in CARS, 2004, pp. 758-763.

[4] D. Selle, B. Preim, A. Schenk, and H.-O. Peitgen, "Analysis of vasculature for liver surgical planning." IEEE Trans. Med. Imaging, vol. 21, no. 11, pp. 1344-1357, Nov 2002.

[5] H. Lang, A. Radtke, M. Hindennach, T. Schroeder, N. R. Frühauf, M. Malago, H. Bourquain, H.-O. Peitgen, K. J. Oldhafer, and C. E. Broelsch, "Impact of virtual tumor resection and computer-assisted risk analysis on operation planning and intraoperative strategy in major hepatic resection," Arch Surg, vol. 140, pp. 629-638, 2005.

[6] W. Lorensen and H. Cline, "Marching cubes: A high resolution 3D surface construction algorithm," $A C M$ Computer Graphics, vol. 21, no. 4, pp. 163-169, Jul 1987.

[7] P. J. Besl and N. D. McKay, "A method for registration of 3-D shapes," IEEE Trans. Pattern Anal. Mach. Intell., vol. 14, no. 2, pp. 239-256, 1992.

[8] M. F. Chen, T. L. Hwang, and C. F. Hung, "Human liver regeneration after major hepatectomy. a study of liver volume by computed tomography." Ann Surg, vol. 213, no. 3, pp. 227-229, Mar 1991.

[9] N. Yamanaka, E. Okamoto, E. Kawamura, T. Kato, T. Oriyama, J. Fujimoto, K. Furukawa, T. Tanaka, F. Tomoda, and W. Tanaka, "Dynamics of normal and injured human liver regeneration after hepatectomy as assessed on the basis of computed tomography and liver function." Hepatology, vol. 18, no. 1, pp. 79-85, Jul 1993.

[10] D. M. Cash, M. I. Miga, T. K. Sinha, R. L. Galloway, and W. C. Chapman, "Compensating for intraoperative soft-tissue deformations using incomplete surface data and finite elements," IEEE Trans. Med. Imaging, vol. 24, no. 11, pp. 1479-1491, Nov. 2005.

[11] L. W. Clements, W. C. Chapman, B. M. Dawant, R. L. Galloway, and M. I. Miga, "Robust surface registration using salient anatomical features for image-guided liver surgery: algorithm and validation." Med Phys, vol. 35, no. 6, pp. 2528-2540, Jun 2008.

[12] L. Clements, P. Dumpuri, W. Chapman, and M. I. Galloway, R. L. Jr. Miga, "Atlas-based method for model updating in image-guided liver surgery," in Medical Imaging 2007: Visualization and Image-Guided Procedures, Proceedings of SPIE, 2007. 